\title{
Dynamic expression of cytokine and transcription factor genes during experimental Fasciola gigantica infection in buffaloes
}

Wei Shi ${ }^{1,2}$, Zhi-Yong Wei ${ }^{1,2}$, Hany M. Elsheikha ${ }^{3}$, Fu-Kai Zhang ${ }^{2}$, Zhao-An Sheng ${ }^{1,2}$, Ke-Jing Lu' ${ }^{1}$, Dong-Ying Wang ${ }^{1,2}$, Wei-Yi Huang ${ }^{1,2^{*}}$ and Xing-Quan Zhu ${ }^{2,4^{*}}$

\begin{abstract}
Background: Determining the mechanisms involved in the immune-pathogenesis of the tropical liver fluke, Fasciola gigantica, is crucial to the development of any effective therapeutic intervention. Here, we examined the differential gene expression of cytokines and transcription factors in the liver of $F$. gigantica-infected buffaloes, over the course of infection.

Methods: Water buffaloes (swamp type) were infected orally with 500 F. gigantica encysted metacercariae. Liver tissue samples were collected 3, 10, 28, 42, 70 and 98 days post-infection (dpi). Levels of gene expression of nine cytokines (IFN- $\gamma$, TGF- $\beta$, IL-1 $\beta$, IL-4, IL-6, IL-10, IL-12B, IL-13 and IL-17A) and four transcription factors (T-bet, GATA-3, Foxp3 and ROR- $-\mathrm{T}$ ) were determined using quantitative real-time PCR (qRT-PCR). We evaluated any correlation between gene expression of these immune-regulatory factors and the severity of liver pathology.

Results: Histopathological examination revealed that cellular infiltration, hemorrhage and fibrosis without calcification in the liver parenchyma of infected buffaloes, increased over the course of infection. This progressive pathology was attributed to dysregulated and excessive inflammatory responses induced by infection. The early infection phase (3-10 dpi) was marked by a generalized immunosuppression and elevated TGF- $\beta$ expression in order to facilitate parasite colonization. A mixed Th1/Th2 immune response was dominant from 28 to $70 \mathrm{dpi}$, to promote parasite survival while minimizing host tissue damage. During late infection (98 dpi), the response was biased towards Th1/Treg in order to inhibit the host's Th2 protective response and promote chronic infection. Both IL-10 and IL-17A and the Th17/Treg balance, played key roles in mediating the inflammatory and immunoregulatory mechanisms in the liver during chronic fasciolosis.

Conclusions: Our data showed distinct $\mathrm{CD}^{+} \mathrm{T}$ helper (Th) polarization and cytokine dysregulation in response to F. gigantica infection in water buffaloes over the course of infection. Characterizing the temporal expression profiles for host immune genes during infection should provide important information for defining how $F$. gigantica adapts and survives in the liver of buffaloes and how host immune responses influence $F$. gigantica pathogenicity.
\end{abstract}

Keywords: Fasciola gigantica, Buffaloes, Liver, Cytokines, Transcription factors, Gene expression

\footnotetext{
* Correspondence: wyhuang@gxu.edu.cn; xingquanzhu1@hotmail.com ${ }^{1}$ College of Animal Science and Technology, Guangxi University, Nanning 530005, Guangxi Zhuang Autonomous Region, People's Republic of China ${ }^{2}$ State Key Laboratory of Veterinary Etiological Biology, Key Laboratory of Veterinary Parasitology of Gansu Province, Lanzhou Veterinary Research Institute, Chinese Academy of Agricultural Sciences, Lanzhou 730046, Gansu Province, People's Republic of China

Full list of author information is available at the end of the article
} the Creative Commons license, and indicate if changes were made. The Creative Commons Public Domain Dedication waiver (http://creativecommons.org/publicdomain/zero/1.0/) applies to the data made available in this article, unless otherwise stated. 


\section{Background}

Fasciolosis is a zoonotic parasitic disease caused by infection with the digenetic trematode flukes of the genus Fasciola. While Fasciola hepatica is prevalent in temperate regions, F. gigantica is more widespread in Africa and Asia [1, 2]. Migration of these flukes inside the body of the host causes severe damage to the liver parenchyma and gall-bladder [3-5]. Buffaloes are economically important animals for the farming communities in developing countries. Infection of buffaloes with F. gigantica is common in southern China and other geographic regions of the world [6]. Infection can cause poor animal health and significant loss of meat and milk production, with considerable financial implications [3, 7]. Fasciola gigantica flukes specifically target the liver of their definitive host. Effective and balanced local immunity is therefore essential for detecting and controlling these hepatotropic parasites, and for limiting hepatic damage.

Liver flukes are, however, efficient immune-modulators and produce many effectors in order to exploit the host immune response to ensure their survival. A recent study in experimentally infected buffaloes reported a modest increase in the level of Th2-type immune cytokines during early $F$. gigantica colonization and immunosuppression during chronic F. gigantica infection [8]. Other studies reported a pro-inflammatory or a mixed Th1/Th2 immune response during early infection, and heightened Th2 and Treg responses during chronic infection. This heightened response was assumed to play roles in restoring the host tissue integrity by damping excessive inflammatory response $[9,10]$. How inflammation contributes to the pathogenesis of F. gigantica is a complex and multifaceted story that is still unfolding.

Hepatic immune-inflammatory mechanisms are essential to maintain liver homeostasis and, if dysregulated (e.g. due to parasite infection), can lead to liver pathology and dysfunction. The abnormal production of cytokines and/ or transcription factors can lead to inadequate control of Fasciola infection [11, 12]. CD4 ${ }^{+}$T-cells are subdivided into Th1, Th2, Th17 and regulatory T-cells (Treg) subsets, based on their pattern of cytokine production [13]. Transcription factors T-bet, GATA-3, Foxp3 and ROR- $\gamma \tau$ play important roles in the differentiation of Th1, Th2, Treg and Th17 cells respectively, and mediate the production of cytokines in these cells [14-16]. Although immunological impairment and polarization of the Th1/Th2 balance are major consequences of $F$. gigantica-induced liver pathology, the expression profile and dynamic changes of Th1/Th2 cytokines during F. gigantica infection has not been completely elucidated. Also, the role of Th17 and Treg cells in the pathogenesis of F. gigantica infection is still not well-defined.

In the present study, we hypothesized that $F$. gigantica infection impairs the balance of Th subsets (Th1/Th2/
Th17) and Treg, thus contributing to the immunepathogenesis of fasciolosis. A temporal study of gene expression of nine cytokines (IFN- $\gamma$, IL-1 $\beta$, IL-12B, IL-4, IL-6, IL-10, IL-13, IL-17A and TGF- $\beta$ ) and four transcription factors (T-bet, GATA-3, Foxp3 and ROR- $\gamma \tau$ ) in the livers from buffaloes infected with $F$. gigantica was conducted using quantitative real-time PCR (qRT-PCR). Data analyses revealed a large number of differentially regulated genes, which exhibited temporal profiles of expression across the time course study. Our results showed evidence of a step-change in gene expression from an 'early' TGF- $\beta$-associated immune-suppersive response (3-10 dpi), to a mixed Th1/Th2 immune response (28-70 dpi) and a 'late' predominantly Th1/Tregdriven response (98 dpi). These results provide new insights into the dynamic immune response of buffaloes to $F$. gigantica over the course of experimental infection.

\section{Methods}

\section{Parasite strain}

Eggs of F. gigantica were obtained from the gall bladder and faeces of naturally infected buffaloes slaughtered for human consumption at local abattoirs (Nanning, Guangxi, P.R. China). Protocols used for the preparation of $F$. gigantica eggs, snail infection with miracidia and harvesting of encysted metacercariae (EM), were performed as previously described [16]. EM were stored in sterile phosphate buffered solution (PBS) at $4{ }^{\circ} \mathrm{C}$. We employed PCR amplification and sequencing of the second internal transcribed spacer (ITS-2) of ribosomal DNA (rDNA) to genotype EM, as described previously [17]. Species identity was confirmed as F. gigantica based on absolute homology to the known ITS-2 sequence of $F$. gigantica from Guangxi province (GenBank: AJ557569). The viability of EM was examined microscopically and only those with viability greater than $90 \%$ were used.

\section{Animals}

Eight to ten month-old (80-100 kg body weight) water buffaloes $(n=35)$ were obtained from a local breeder and were identified as swamp type by karyotypic analysis. Buffaloes were kept in separate concrete floor pens. Commercial feed and clean water were provided ad libitum for all animals during the entire study period. None of the buffaloes had been used previously for any experimental procedure. Animals were confirmed as negative in terms of prior infection with liver flukes, by negative fecal examination and negative serum $F$. gigantica-specific IgG-antibody-based ELISA prior to the start of the study. All animals were treated with a single dose of triclabendazole $(5 \% \mathrm{w} / \mathrm{v})$ in order to eliminate any potential existing fluke infection that may have been missed on laboratory examination. Following triclabendazole 
treatment, buffaloes were allowed to acclimatize for 30 days to avoid any residual efficacy of the treatment on the establishment of experimental F. gigantica infection.

\section{Animal inoculation and tissue collection}

Thirty-five buffaloes were assigned randomly to seven different groups (5 buffaloes/group). Group I was composed of 5 buffaloes that were mock-incoulated with PBS only. Buffaloes in Groups II-VII were each infected with 500 viable metacercariae by oral gavage. Control buffaloes were euthanized at the start of the experiment to obtain baseline values for hepatic tissue pathology and gene expression. Animals from each of the six infected groups were sacrificed and their livers were harvested at $3,10,28,42,70$ and 98 days post infection (dpi), for histopathological and molecular studies. Group III-VII buffaloes were examined clinically on a weekly basis for the development of clinical signs of fasciolosis. The control group served as a baseline point of reference for monitoring the progressive changes in gene expression over the course of infection. However, the inclusion of matched control groups receiving placebo (treated with vehicle only) and euthanized at the same time points as infected groups would have strengthened the power of the study. Alternatively, control buffaloes could have been kept alive (rather than killing them at the start) and liver punch biopsy samples obtained from them at each of the above time points. Despite the early sacrifice of the control animals for reasons of economy and resources, we believe the effects we have observed to be due to the experimental manipulation.

\section{Gross examination and histopathological evaluation}

At six time points after infection (indicated above), animals in each infected group were sacrificed, their livers were harvested and examined for pathological lesions and the presence of the flukes. Parasite eggs were recovered by filtering bile fluid through a $0.15 \mathrm{~mm}$ pore size mesh. Fasciola gigantica infection was confirmed by observing gross pathological lesions, associated with flukes in the livers and/or by the presence of flukes and eggs in the bile ducts. Samples of liver tissue $(\sim 8 \mathrm{~g})$ showing pathological lesions were collected from each animal. Tissue samples were resuspended in 10\% PBS-buffered formalin solution overnight, then dehydrated in alcohol, rinsed in xylene, and embedded in paraffin. $3 \mu \mathrm{m} \mathrm{sec-}$ tions of paraffin-embedded tissue were mounted onto glass slides, and stained with hematoxylin and eosin (H\&E). Stained tissue sections were examined microscopically at 400× magnification and imaged using a Zeiss Axio Imager manual upright research microscope. Additional liver tissue samples for RNA extraction were collected and kept in RNA store buffer (Tiangen
Biotech, Beijing, China), snap frozen in liquid nitrogen and stored at $-80{ }^{\circ} \mathrm{C}$.

\section{RNA isolation}

Total RNA was extracted from frozen liver tissue samples by RNAprep Pure Tissue Kit (Tiangen Biotech, Beijing, China) following the manufacturer's instructions. RNA integrity was examined by $2 \%$ agarose gel electrophoresis and quantified by NanoDrop 2000/2000c Spectrophotometer analysis (Thermo Scientific, Waltham, US).

\section{Quantification of cytokine and transcription factor gene expression}

Quantitative gene expression analysis was performed on liver samples obtained from uninfected control animals, and from infected animals on 3, 10, 28, 42, 70 and 98 dpi. Levels of mRNA expression of nine cytokines (IFN$\gamma$, IL-1 $\beta$, IL-12B, IL-4, IL-6, IL-10, IL-13, IL-17A and TGF- $\beta$ ) and four transcription factors (T-bet, GATA-3, Foxp3 and ROR- $\gamma \tau$ ) were determined using quantitative real-time PCR (qRT-PCR). All qRT-PCR primers used in the study are described in Table 1. Complementary DNA (cDNA) was synthesized from 500 ng RNA samples using a PrimerScript ${ }^{\mathrm{TM}} \mathrm{RT}$ reagent kit (TaKaRa Bio, Dalian, China). qRT-PCR was performed using SYBR ${ }^{\odot}$ Premix Ex Taq ${ }^{\text {Tx }}$ II (Tli RNaseH Plus, TaKaRa Bio) and a CFX96 real-time PCR instrument (Bio-Rad, Hercules, US). To determine the specificity of amplification, melting curve analysis was applied to all final PCR products. The efficiency of qRT-PCR, and relative quantification $(R Q)$ of gene expression, were analyzed using the comparative $2^{-\Delta \Delta C q}$ method [18]. The level of expression of each gene was normalized using glyceraldehyde-3phosphate dehydrogenase $(\mathrm{GAPDH})$ as the reference housekeeping gene.

\section{Data analysis}

Statistical analysis and graph production were performed using GraphPad Prism (GraphPad Software Inc., La Jolla, CA, USA, version 6.02.). Levels of cytokine and transcription factor mRNA expression between uninfected and infected groups were compared at different time points after infection using one-way analysis of variance (ANOVA) with post-hoc LSD multiple comparison tests. Results were presented as $F_{(\mathrm{DFn}, \mathrm{DFd})}$ and $P$-value. Pearson's correlation coefficients ( $r$-value) were used to detect any correlation between the measured level of Th1, Th2, Treg and Th17 immune cytokines and transcription factors (T-bet, GATA-3, Foxp3 and ROR- $\gamma \tau$ ) gene expression between infected groups, followed by a twotailed post-hoc test and presented as a $P$-value. Data shown represent the mean \pm SEM of results from five buffaloes. The level of significance for all analyses was evaluated with a confidence interval $>95 \%(P<0.05)$. 
Table 1 List of primers used in the SYBR green-based qRT-PCR analysis

\begin{tabular}{|c|c|c|c|c|}
\hline Gene target & & Primer sequence $\left(5^{\prime}-3^{\prime}\right)$ & Product length (bp) & Reference \\
\hline \multirow[t]{2}{*}{ GAPDH } & $\mathrm{F}$ & CCTGCACCACCAACTGCTTG & 222 & {$[62]$} \\
\hline & $\mathrm{R}$ & TTGAGCTCAGGGATGACCTTG & & \\
\hline \multirow[t]{2}{*}{$\mathrm{IFN}-\gamma$} & $\mathrm{F}$ & GTCTCCTTCTACTTCAAACT & 253 & {$[63]$} \\
\hline & $\mathrm{R}$ & ATTCTGACTTCTCTTCCGCT & & \\
\hline \multirow[t]{2}{*}{ TGF- $\beta$} & $\mathrm{F}$ & CGTGCTAATGGTGGAATAC & 208 & Present study \\
\hline & $\mathrm{R}$ & GCCAGGAATTGTTGCTATA & & \\
\hline \multirow[t]{2}{*}{$\mathbb{I L}-1 \beta$} & $\mathrm{F}$ & CTAGCCCATGTGTGCTGAAG & 59 & {$[62]$} \\
\hline & $\mathrm{R}$ & CCTTTACTTGGCTCTTCACC & & \\
\hline \multirow[t]{2}{*}{$\mid \mathrm{L}-4$} & $\mathrm{~F}$ & CAGCATGGAGCTGCCT & 177 & {$[64]$} \\
\hline & $\mathrm{R}$ & ACAGAACAGGTCTTGCTTGC & & \\
\hline \multirow[t]{2}{*}{ IL-6 } & $\mathrm{F}$ & CTGCAATGAGAAAGGAGATA & 191 & {$[63]$} \\
\hline & $\mathrm{R}$ & GGTAGTCCAGGTATATCTGA & & \\
\hline \multirow[t]{2}{*}{ IL-10 } & $\mathrm{F}$ & CTGTGCCTCTCCCCTAGAGT & 236 & [62] \\
\hline & $\mathrm{R}$ & GCAGCTAGCTCCACAAGGAA & & \\
\hline \multirow[t]{2}{*}{ IL-12B } & $\mathrm{F}$ & CAGGGACATCATCAAACCAG & 213 & {$[63]$} \\
\hline & $\mathrm{R}$ & CTTGTGGCATGT GACTITGG & & \\
\hline \multirow[t]{2}{*}{$\mathbb{I L}-13$} & $\mathrm{~F}$ & AGAACCAGAAGGTGCCGCT & 50 & {$[65]$} \\
\hline & $\mathrm{R}$ & GGTTGAGGCTCCACACCATG & & \\
\hline \multirow[t]{2}{*}{ IL-17A } & $\mathrm{F}$ & CTACAGTGAACTGGAAGGAAC & 554 & Present study \\
\hline & $\mathrm{R}$ & AAAAGGGGCTGGGTCT & & \\
\hline \multirow[t]{2}{*}{ T-bet } & $\mathrm{F}$ & CCTGGACCCAACTGTCAACT & 171 & [66] \\
\hline & $\mathrm{R}$ & GAAACTCGGCCTCATAGCTG & & \\
\hline \multirow[t]{2}{*}{ GATA-3 } & $\mathrm{F}$ & GATCAAGCCCAAGCGAAGG & 124 & Present study \\
\hline & $\mathrm{R}$ & CCGCAGGCATTGCAGACA & & \\
\hline \multirow[t]{2}{*}{ Foxp3 } & $\mathrm{F}$ & GACAGCACCCTTTCGACTGT & 191 & {$[66]$} \\
\hline & $\mathrm{R}$ & CTCCAGAGATTGCACCACCT & & \\
\hline \multirow[t]{2}{*}{$R O R-\gamma \tau$} & $\mathrm{F}$ & CTACAGTGAACTGGAAGGAAC & 554 & Present study \\
\hline & $\mathrm{R}$ & AAAAGGGGCTGGGTCT & & \\
\hline
\end{tabular}

Abbreviations: $F$ forward primer, $R$ reverse primer

\section{Results}

Gross and histopathological attributes

Even though buffaloes did not exhibit clear clinical signs, $F$. gigantica induced a wide range of pathological lesions over the course of infection (Table 2). During the early stage of infection (3-70 dpi), immature flukes migrated through the intestinal wall, abdominal cavity, liver capsule and liver parenchyma, to reach the bile ducts where they attain sexual maturity. Migration of the juvenile flukes through the host's tissues was characterized by classical inflammatory signs and accumulation of fibroblasts towards the end of this stage, but without obvious fibrosis. The total number (and average length) of flukes recovered from the livers of infected groups at 28, 42, 70 and $98 \mathrm{dpi}$ were: 3 flukes $(1.5 \mathrm{~mm}), 22$ flukes $(2.5 \mathrm{~mm})$, 65 flukes $(8 \mathrm{~mm})$, and 36 flukes $(14 \mathrm{~mm})$, respectively. No fluke was observed in the liver or bile duct before 28 dpi, probably because they were too small to be visible to the naked-eye. Hepatic hemorrhage, swelling, necrosis and viscous bile were first detected at $10 \mathrm{dpi}$. Tissue fibrosis was observed at $70 \mathrm{dpi}$, along with disappearance of hemorrhage. Seven parameters were used to describe the histopathological features in buffalo livers during infection (Table 3). Histopathological characteristics of the infected liver tissues (Fig. 1) included an infiltration of inflammatory cells, such as neutrophils and lymphocytes at $3 \mathrm{dpi}$. As infection progressed, an accumulation of eosinophils, monocytes and red blood cells (RBCs) was detected (10-28 dpi), while fibroblast formation and bile duct hyperplasia were observed later (42 dpi). During the chronic stage (70-98 dpi), significant fibrosis and necrosis, without calcification, were observed. Fluke eggs were detected in the bile and feces of infected animals at 98 dpi. Granulomas with necrotic centers, 
Table 2 The presence (+) and absence (-) of gross pathological lesions, flukes and fluke eggs in the liver of buffaloes experimentally infected with F. gigantica

\begin{tabular}{|c|c|c|c|c|c|c|c|c|c|c|c|}
\hline Group & Dpi & No. of animals & Hemorrhage & Swelling & Parasite tunnel & Fibrosis & Necrosis & Bile duct hyperplasia & Viscous bile & Visible flukes & Eggs \\
\hline I & 0 & 5 & - & - & - & - & - & & - & - & - \\
\hline$\|$ & 3 & 5 & - & - & - & - & - & - & - & - & - \\
\hline III & 10 & 5 & + & + & - & - & + & - & + & - & - \\
\hline IV & 28 & 5 & + & + & - & - & + & - & + & + & - \\
\hline V & 42 & 5 & - & + & + & - & + & + & + & + & - \\
\hline $\mathrm{Vl}$ & 70 & 5 & - & + & + & + & + & + & + & + & - \\
\hline VII & 98 & $4^{\mathrm{a}}$ & - & + & + & + & + & + & + & + & + \\
\hline
\end{tabular}

aNA from one animal in this group VII did not pass the quality control check and was therefore excluded from the analysis

heavy infiltrates of lymphocytes, RBCs and eosinophils were present at $98 \mathrm{dpi}$.

\section{Gene expression of cytokines}

To determine how immune response changed over the course of infection, gene expression of nine cytokines was assessed in the liver tissue of 29 infected buffaloes and five uninfected buffaloes using qRT-PCR. RNA from one animal from group VII did not pass the quality control check and was therefore excluded from the analysis. Results showed that infection had a significant impact on gene expression of Th1, Th2, Th17, and Treg cytokines (Fig. 2a and Table 4). The transcriptional profile of cytokine genes showed an immunosuppressive state during early infection, a mixed Th1/Th2 response as the infection progressed, and a shifting to Th1/Treg response, associated with greater histopathological changes and fibrosis during late stage infection.

The gene expression of Th1 cytokines (IFN- $\gamma$ and IL12B) and IL-10 showed no significant change during early infection. IFN- $\gamma$ showed significantly higher expression at $70 \mathrm{dpi}(P=0.0079)$ and $98 \mathrm{dpi}(P=0.0003)$, when compared with the control. Relative gene expression of both of IL-12B $(P<0.0001)$ and IL-10 $(P=$ $0.0036)$ peaked at $98 \mathrm{dpi}$. Fluke infection also upregulated IL-4 expression at $28 \mathrm{dpi}(P=0.0028)$, whereas IL13 significantly increased at $28 \mathrm{dpi}(P=0.0259)$ and 70 dpi $(P=0.0426)$, peaking at $98 \mathrm{dpi}(P<0.0001)$. TGF- $\beta$
mRNA was rapidly elevated at 3 dpi $(P=0.0003)$, followed by a decrease to the basal level and remained low thereafter. Upregulation of IL-17A was noted at 28 dpi $(P=0.0114)$, however expression was relatively low based on a high $C q$ value, suggesting it to be of little diagnostic value as a marker for Th17 cytokine in this study. The kinetics of the cytokines IL- $1 \beta$ and IL-6 mRNA were similar, both peaking at $28 \mathrm{dpi}(P=0.0007$ for IL-1 $\beta ; P=0.0002$ for IL-6) and $98 \mathrm{dpi}(P<0.0001$ for IL-1 $\beta ; P=0.0008$ for IL-6).

Spearman's correlation analysis between each pair of cytokines was performed based on the $R Q$ values from infected groups (Fig. 3a). This analysis revealed a significant positive correlation between the expression of IL-12B mRNA and Th2 cytokines (IL-4: $r_{(29)}=0.44, P=$ 0.0336; IL-13: $\left.r_{(29)}=0.41, P=0.0542\right)$, and between IFN$\gamma$ and IL-12B $\left(r_{(29)}=0.62, P=0.0017\right)$ or IL-13 $\left(r_{(29)}=\right.$ $0.51, P=0.0124)$. The gene expression of IL-10 was significantly correlated with both Th1 and Th2 cytokines (IFN- $\gamma: r_{(29)}=0.49, P=0.0171$; IL-12B: $r_{(29)}=0.50, P=$ 0.014; IL-4: $r_{(29)}=0.80, P<0.0001$; IL-13: $r_{(29)}=0.51, P=$ $0.014)$ and with IL-17A $\left(r_{(29)}=0.73, P<0.0001\right)$. Positive correlation was also found between IL-4 and IL-13 $\left(r_{(29)}\right.$ $=0.44, P=0.0336)$ gene expression. There was only a statistically significant relationship between IL-17A expression and IL-4 $\left.r_{(29)}=0.87, P<0.0001\right)$ and IL-10 $\left(r_{(29)}=0.73, P<0.0001\right)$, but not with any of the other cytokines. Although not significant, the relative mRNA

Table 3 The presence (+) and absence (-) of the histopathological changes observed in the liver of buffaloes experimentally infected with F. gigantica

\begin{tabular}{|c|c|c|c|c|c|c|c|c|c|}
\hline Group & Dpi & No. of animals & Inflammatory infiltration & RBCs & Eosinophils & Neutrophils & Fibroblasts & Lymphocytes & Monocytes \\
\hline I & 0 & 5 & - & - & - & - & - & - & - \\
\hline$\|$ & 3 & 5 & + & - & - & + & - & + & - \\
\hline III & 10 & 5 & + & + & + & + & - & + & - \\
\hline IV & 28 & 5 & + & + & + & + & - & + & + \\
\hline V & 42 & 5 & + & + & + & + & + & + & + \\
\hline $\mathrm{Vl}$ & 70 & 5 & + & + & + & + & + & + & + \\
\hline VII & 98 & 4 & + & + & + & + & + & + & + \\
\hline
\end{tabular}




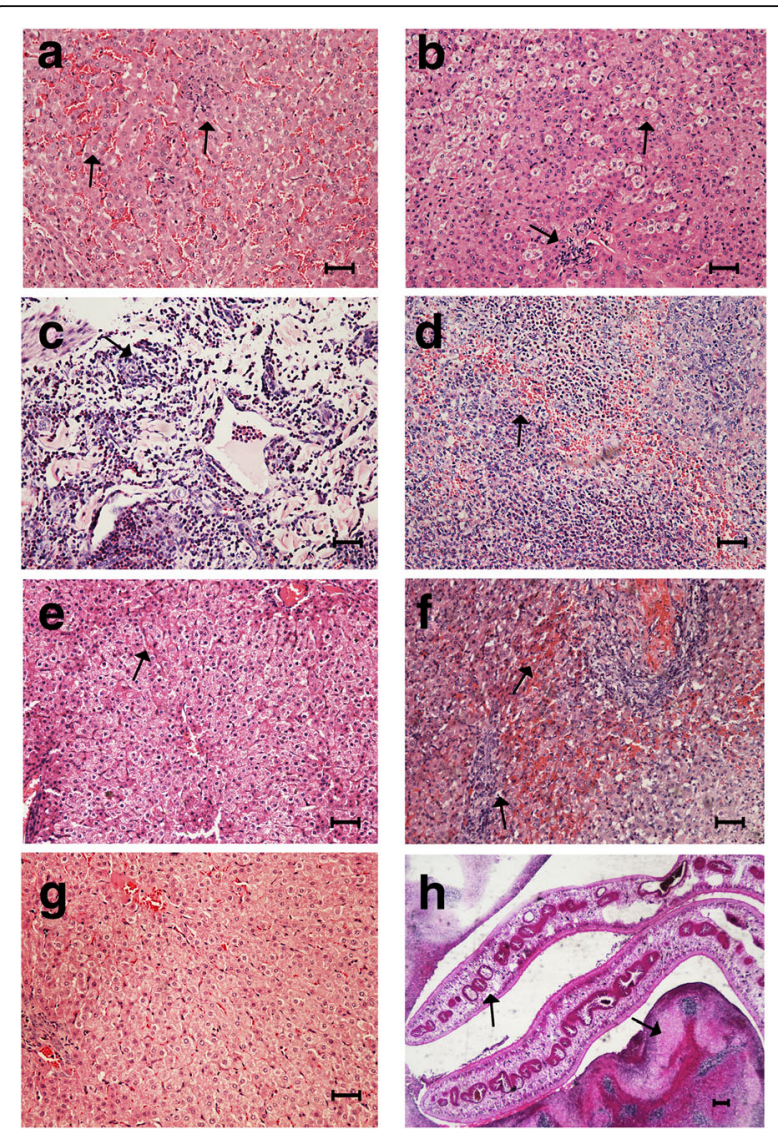

Fig. 1 Histopathological characteristics of the livers of buffaloes infected with Fasciola gigantica. a At 3 dpi, there was local hyperemia associated with mild filtration of lymphocytes and neutrophils. b At 10 $\mathrm{dpi}$, there was scattered vacuolation of hepatocytes, consistent with fat, along with mild to moderate focal necrosis. c At 28 dpi, diffuse intravascular coagulation, severe infiltration of inflammatory cells mainly neutrophils and lymphocytes, and granular degeneration of the cytoplasm were observed. d At 42 dpi, moderate to severe multifocal hemorrhages and necrosis, infiltration of eosinophils, RBCs and monocytes, and accumulation of fibroblasts were detected. e At $70 \mathrm{dpi}$, there were mild to moderate multifocal bile duct hyperplasia and focal coagulative necrosis associated with collagen deposition. $\mathbf{f}$ At 98 dpi, severe periportal fibrosis associated with multifocal inflammatory infiltrate, cellular debris, moderate multifocal hemorrhages, and granulomas with necrotic centres were detected. $\mathbf{g}$ Liver tissue from uninfected animal showed normal histological architecture of the hepatic tissue. $\mathbf{h}$ Adult flukes in the intrahepatic bile duct, along with epithelial hyperplasia of the duct. In all figures, tissue sections were stained with $\mathrm{H} \& \mathrm{E}$ and arrows point at the corresponding morphological features described above. Scale-bars: a-g, $50 \mu \mathrm{m} ; \mathbf{h}, 100 \mu \mathrm{m}$

expression of TGF- $\beta$ showed an inverse relationship to any other cytokine.

\section{Expression profiles of transcription factors}

Fasciola gigantica infection altered the expression of $\mathrm{T}$ bet, GATA-3 and ROR- $\gamma \tau$, with the expression of Foxp3 being least affected (Fig. 2b and Table 4). Compared with the control, mRNA of T-bet was highly expressed at 98 dpi $(P=0.0113)$, while the expression of the GATA-3, Foxp3 and ROR- $\gamma \tau$ genes was upregulated at $28 \mathrm{dpi}(P=$ $0.0035, P=0.0424$ and $P=0.0002$, respectively). Spearman's correlation analysis indicated that the expression of T-bet and Foxp3 was correlated $\left(r_{(29)}=0.63, P=\right.$ 0.0011). Positive correlation was also found between the expression of ROR- $\gamma \tau$ and GATA-3 $\left(r_{(29)}=0.43, P=\right.$ $0.039)$ or Foxp3 $\left(r_{(29)}=0.42, P=0.0439\right)$ (Fig. 3b). As shown in Fig. 3c, the mRNA expression of GATA-3 correlated significantly with that of IL-4 $\left(r_{(29)}=0.54, P=\right.$ 0.0072 ), but was less correlated with another Th2 cytokine IL-13 $\left(r_{(29)}=0.017, P=0.9387\right)$, whereas, the expression of ROR- $\gamma \tau$ was correlated with that of IL-17A $\left(r_{(29)}=0.54, P=0.0072\right)$. There was a tendency of correlation between the expression of T-bet and Th1 cytokine IFN- $\gamma\left(r_{(29)}=0.22, P=0.305\right)$ or IL-12B $\left(r_{(29)}=0.33, P=\right.$ $0.1265)$ and between the expression of Foxp3 and IL-10 $\left(r_{(29)}=0.38, P=0.0718\right)$.

\section{Th1/Th2/Th17/Treg balance}

To better understand the temporal trends in gene expression of Th1, Th2, Th17 and Treg in the liver postinfection, the ratios between the expression of transcription factors were evaluated using the pairwise comparison method (Fig. 2c). The $R Q$ ratio of T-bet/GATA-3 was employed as an indicator of Th1/Th2 cytokine gene expression pattern. This ratio was higher in the liver at 98 dpi when compared to the uninfected group $(P=0.0049)$. This analysis also revealed that Th1/Th17 $(P=0.0246)$ and Treg/Th17 $(P=0.0126)$ were both upregulated at 98 $\mathrm{dpi}$, when compared with the samples tested at other time points (3-70 dpi). However, no significant change was found in either Th1/Treg, Th2/Treg, or Th2/Th17 expression ratios at any of the examined time points. These data suggest a predominance of the Th1 and Treg cellular immune response at $98 \mathrm{dpi}$.

\section{Discussion}

Our findings provide an increased understanding of the immuno-inflammatory response of buffaloes to $F$. gigantica infection through examining the correlation between gene expression of nine cytokines and four transcription factors in Th1, Th2, Th17 and Treg cells, together with the liver pathology during early, mid and late stages of infection. We initially characterized gross and histopathological changes in the liver at six time points following infection compared to uninfected control. Although infected buffaloes did not exhibit clinical signs, they developed hepatic gross pathologies similar to what have been observed in other studies [15, 19]. Histopathological features also agreed with previous studies on $F$. gigantica $[9,20,21]$ and F. hepatica [22]. An unexpected finding was that none of the examined liver from infected animals showed calcium 


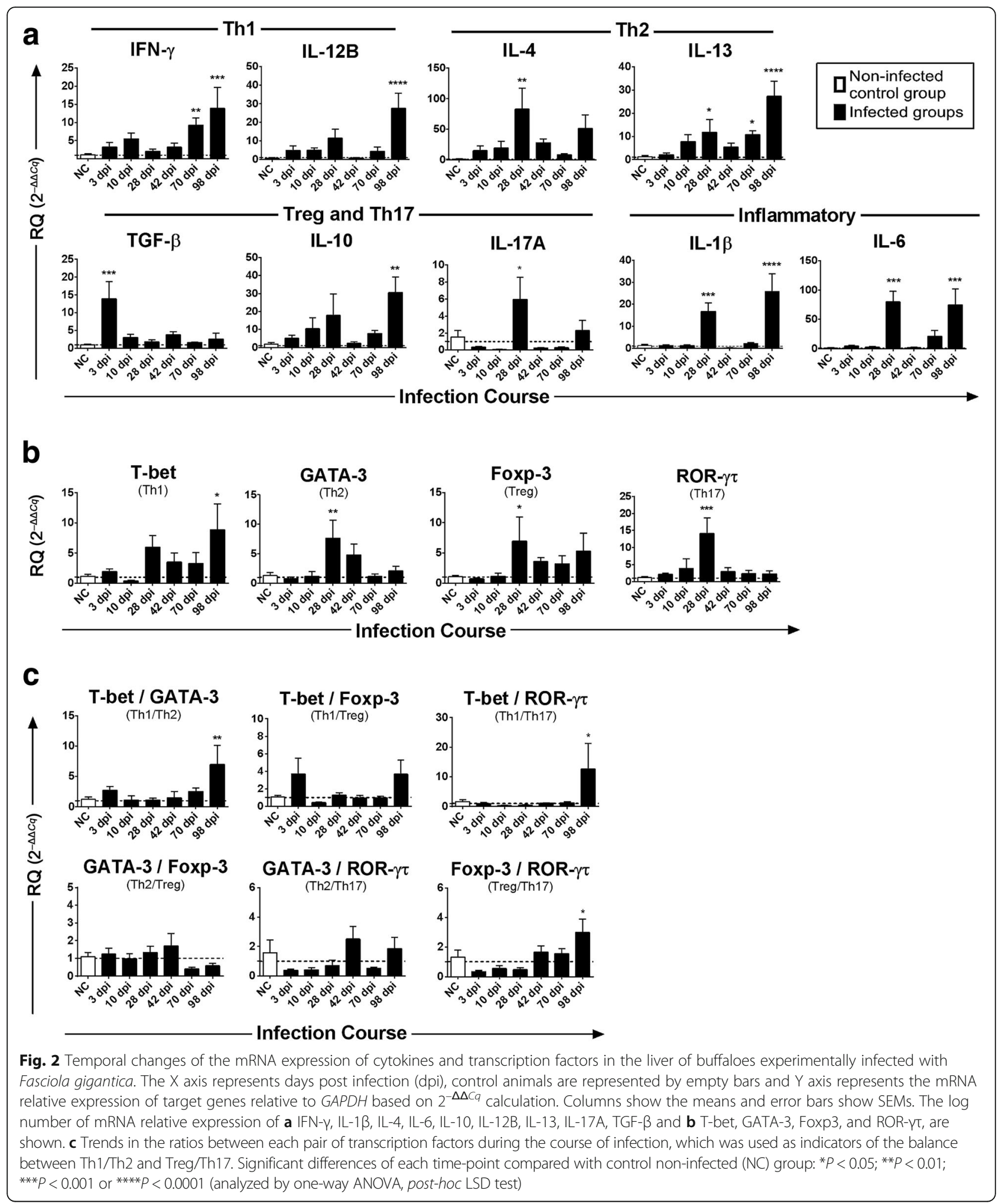

deposits. The difference in liver calcification between our study and previous reports may relate to variations in the experimental conditions, such as using different infectious doses, different parasite strains, the permissibility of the host species, the duration of the experimental infection, and using single infection vs repeated infections [21-23].

Next, we have shown that buffaloes showed a substantial liver immune response subsequent to oral challenge with 
Table 4 Relative gene expression in the liver of buffaloes experimentally infected with $F$. gigantica compared to the control group. The relative quantities were obtained according to the comparative $C q$ method $\left(2^{-\Delta \Delta C q}\right)$. $P$ values indicate statistical significance $(P<0.05)$

\begin{tabular}{|c|c|c|c|c|c|c|c|c|c|c|c|c|c|c|}
\hline \multirow{3}{*}{$\begin{array}{l}\text { Gene } \\
\text { Target }\end{array}$} & \multirow{2}{*}{\multicolumn{2}{|c|}{ ANOVA }} & \multicolumn{12}{|c|}{ Multiple comparisons } \\
\hline & & & \multicolumn{2}{|l|}{$3 \mathrm{dpi}$} & \multicolumn{2}{|c|}{$10 \mathrm{dpi}$} & \multicolumn{2}{|l|}{$28 \mathrm{dpi}$} & \multicolumn{2}{|c|}{$42 \mathrm{dpi}$} & \multicolumn{2}{|c|}{$70 \mathrm{dpi}$} & \multicolumn{2}{|l|}{$98 \mathrm{dpi}$} \\
\hline & $F_{(\mathrm{DFn}, \mathrm{DFd})}$ & $P$ & $R Q$ & $P$ & $R Q$ & $P$ & $R Q$ & $P$ & $R Q$ & $P$ & $R Q$ & $P$ & $R Q$ & $P$ \\
\hline $\mathrm{IFN}-\gamma$ & $4.693_{(6,21)}$ & 0.0035 & 2.08 & 0.4326 & 5.51 & 0.1282 & 2.06 & 0.7358 & 3.21 & 0.4590 & 9.27 & 0.0079 & 13.91 & 0.0003 \\
\hline TGF- $\beta$ & $4.738_{(6,19)}$ & 0.0041 & 13.93 & 0.0003 & 3.07 & 0.4884 & 1.67 & 0.8036 & 3.85 & 0.3408 & 1.63 & 0.8371 & 2.66 & 0.6062 \\
\hline $\mid L-1 \beta$ & $11.05_{(6,26)}$ & $<0.0001$ & 1.31 & 0.9767 & 1.27 & 0.9811 & 14.25 & 0.0007 & 0.26 & 0.8137 & 2.31 & 0.8025 & 28.45 & $<0.000$ \\
\hline $\mid \mathrm{L}-4$ & $2.877_{(6,24)}$ & 0.0291 & 7.01 & 0.5847 & 19.4 & 0.4892 & 97.21 & 0.0028 & 27.9 & 0.3121 & 9.43 & 0.7749 & 67.14 & 0.0630 \\
\hline IL-6 & 7.636 & 0.0001 & 5.50 & 0.8529 & 2.81 & 0.9366 & 78.1 & 0.0002 & 1.94 & 0.9728 & 23.14 & 0.2805 & 94.56 & 0.0008 \\
\hline IL-10 & $2.514_{(6,26)}$ & 0.0471 & 5.99 & 0.7003 & 9.92 & 0.3184 & 21.18 & 0.0694 & 2.3 & 0.9579 & 6.24 & 0.4902 & 32.67 & 0.0036 \\
\hline$L-12 B$ & $5.595_{(6,26)}$ & 0.0008 & 5.92 & 0.4786 & 3.99 & 0.4746 & 13.68 & 0.0759 & 0.86 & 0.9825 & 5.37 & 0.5312 & 24.84 & $<0000$ \\
\hline IL-13 & $6.56_{(6,20)}$ & 0.0006 & 2.09 & 0.8459 & 7.80 & 0.1522 & 11.87 & 0.0259 & 5.45 & 0.3499 & 10.8 & 0.0426 & 27.33 & $<0.000$ \\
\hline$L-17 A$ & $3.532_{(6,21)}$ & 0.0142 & 0.33 & 0.4612 & 0.07 & 0.3694 & 5.95 & 0.0114 & 0.26 & 0.4327 & 0.28 & 0.4400 & 2.99 & 0.6354 \\
\hline T-bet & $2.173_{(6,28)}$ & 0.076 & 2.16 & 0.7923 & 0.25 & 0.7830 & 4.73 & 0.1018 & 3.93 & 0.4158 & 3.99 & 0.4606 & 11.38 & 0.0113 \\
\hline GATA-3 & $3.319_{(6,28)}$ & 0.0135 & 0.84 & 0.8101 & 1.42 & 0.9579 & 5.18 & 0.0035 & 4.80 & 0.0889 & 1.34 & 0.9397 & 1.8 & 0.6952 \\
\hline Foxp3 & $1.455_{(6,28)}$ & 0.2296 & 0.70 & 0.9054 & 1.07 & 0.9749 & 3.03 & 0.0424 & 3.97 & 0.3705 & 3.40 & 0.4458 & 3.05 & 0.1372 \\
\hline$D R-\gamma \tau$ & $4.387_{(6,28)}$ & 0.003 & 2.25 & 0.7567 & 4.57 & 0.3780 & 10.28 & 0.0002 & 3.24 & 0.5637 & 2.68 & 0.7076 & 2.55 & 0.7148 \\
\hline
\end{tabular}

Abbreviations: $R Q$, relative quantity (mean); $F_{(\mathrm{DFn}, \mathrm{DFd})}$, degrees of freedom with numerator and degrees of freedom denominator inside the subscript parentheses

500 F. gigantica encysted metacercariae. Certain genes exhibited shifting temporal expression patterns as the infection proceeded. Expression of cytokine and transcription factor genes were decreased at 3 and $10 \mathrm{dpi}$, suggesting a general immunosuppression state to allow parasite colonization. Cytokine reduction during the early stage of F. gigantica infection has been reported previously [24, 25]. A previous study has documented the downregulation of MHC-II related genes, as well as the suppression of the host pro-inflammatory (Th1) immune response during early $F$. gigantica infection [16]. This immunosuppression might be attributed to an increased expression of the immunosuppressive cytokine TGF- $\beta$. While TGF- $\beta$ was upregulated at $3 \mathrm{dpi}$, Foxp3 (Treg transcription factor) was upregulated at a later stage of infection (28 dpi), indicating that Tregs (also known as Foxp3-expressing cells) play a more diverse role than causing early immunosuppression in regulating immune responses. Although increased TGF- $\beta$ at 3 dpi may have promoted the generation of Foxp3-expressing Tregs, increased IL-6 at 28 dpi may have abrogated the suppressive function of Tregs [26] by antagonizing TGF- $\beta$-induced Treg generation and stimulating Treg differentiation to Th17, as reported previously [27].

The inflammatory cascades triggered by $F$. gigantica must be tightly coordinated in order to avoid severe liver pathology. Th2 cells mediate humoral responses via induction of IL-4, IL-5, IL-9, IL-10 and IL-13 cytokines, which are important for controlling extracellular parasites [28]. In agreement with this, our results at $28 \mathrm{dpi}$ showed a significant increase in the expression of the anti-inflammatory Th2-associated cytokines (IL-4, IL-13) and transcription factor (GATA-3), indicating the induction of Th2 response in liver tissue, probably to limit the fluke development and to balance the increased levels of inflammatory cytokines IL-1 $\beta$, IL- 6 and IL-17A. This response was expected because IL- 4 is known to have the greatest effect in inducing Th2 differentiation [29] and the GATA-3 transcription factor is a key regulator of Th2 differentiation [30]. IL-4 stimulation may have activated STAT6, which is the major signal transducer in IL-4-mediated Th2 differentiation [31]. STAT6 is known to promote Th2 differentiation by stimulating GATA-3 [32]. Therefore, this coordinated response may be mediated by the parasite and/or the host in order to promote parasite survival while minimizing host hepatic damage. Th2-predominant response together with the suppression of Th1/Th17 response has been reported previously [33-36]. Interestingly, some molecules secreted by Fasciola species can suppress the differentiation of Th17 cells independently of Th2 cells differentiation, by altering the function of dendritic cells [37].

Although $\mathrm{CD} 4^{+}$Th17 cells play a role in the control of a variety of parasitic infections, the role of IL-17 produced by $\mathrm{CD}^{+}$Th17 cells in immunity to $F$. gignatica has not been clearly defined [38, 39]. Our current and previous studies [8], demostrated that IL-17 may play a role in the inflammatory process during early $F$. gigantica infection. Th17 cells are a newly-identified class of effector T cell, which produces IL-17A and IL-17F. Th17 cells can contribute to resistance to many intracellular [40] and extracellular parasites [8]. In addition to controlling infection, IL-17 expression has been associated with inflammatory and allergic responses [41]. TGF- $\beta$ and IL-6 act cooperatively and non-redundantly to 


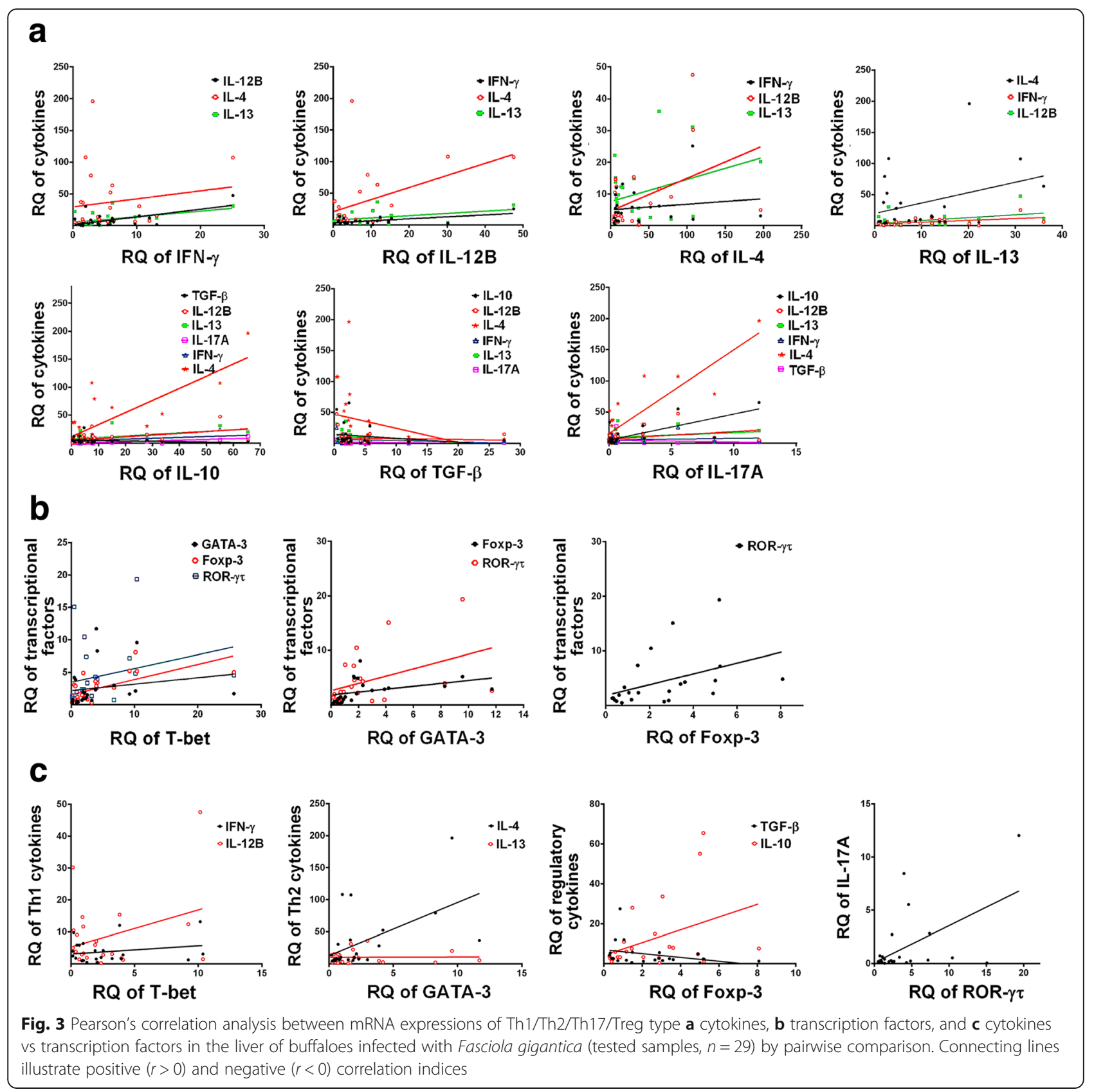

promote Th17 activity [27]. In agreement with this, TGF- $\beta$ was upregulated at $3 \mathrm{dpi}$, and both IL- 6 and IL$17 \mathrm{~A}$ were upregulated at $28 \mathrm{dpi}$. IL-6 was found to be important in suppressing Treg generation in order to promote Th17 differentiation as discussed above. The expression of retinoic acid-related orphan receptors ROR- $\gamma \mathrm{t}$, a key transcription factor in Th17 differentiation $[42,43]$, was positively correlated with the $R Q$ level of IL-17A at $28 \mathrm{dpi}$.

At 70 and 98 dpi a mixed Th1/Th2 type profile, supported by co-dominance of IFN- $\gamma$, IL-1 $\beta$, IL-12B, IL-6, IL-4 and IL-13 cytokines, was observed as consistent with the result obtained by Kumar [44]. The high expression of Th1 cytokines (IL-1 $\beta$ and IL-6), suggesting acute inflammatory activity, correlated with hemorrhage, necrosis and severe infiltration of inflammatory cells in the liver. Th2 cytokines, on the other hand, often lead to chronic inflammatory response by promoting fibrosis and tissue remodeling $[45,46]$. The accumulation of fibroblasts during chronic infection suggests that Th2 cytokine-mediated tissue repair was taking place. This state of immune homeostasis is probably required for parasite persistence and was correlated with an increased number of flukes. The T-bet/GATA-3 ratio, a 
marker of Th1/Th2 [47], was not altered during acute infection, but was significantly increased when the infection became chronic, suggesting more bias towards a Th1-mediated inflammatory response during late infection. This observation contradicts previous studies in mice, cattle and buffaloes infected with F. gigantica that reported a mixed Th1/Th2 response with a predominance of a Th2-biased pattern [48, 49]. Other studies have reported a Th2 response in early infection and an increased Th0-type response during chronic F. gigantica infection in cattle [35] and immunosuppression during chronic F. gigantica infection in buffaloes [8]. These differing findings between studies may result from different experimental conditions.

A synergism between IL-1 $\beta$ and IL-17 has previously been reported [50]. In our study, a strong correlation was observed between IL-17A and IL-4 and IL-10, as well as between ROR- $\gamma \tau$ and GATA-3 and Foxp3, implying that induction of Th17 was paralleled by induction of both Th2 and Treg. This refutes the antagonistic relationship previously reported between a polarized Th2/ Treg immune response and suppression of Th1/Th17 cytokines [11, 51-53]. A gradual upregulation of IL-10 mRNA expression was observed during early infection, in agreement with our previous finding in buffalo's serum [8]. IL-10 levels significantly increased at $98 \mathrm{dpi}$, which was also reported in a study on $F$. hepatica-infected sheep [54]. IL-10 is considered to be a regulatory cytokine rather than a Th2 type cytokine $[55,56]$. High levels of IL-10 can suppress excessive inflammation induced by the parasite $[57,58]$. In our study, elevated levels of IL-10 and the concurrent upregulation of Th1 and Th2 cytokine genes in the liver suggest that IL-10 was required for the Th2 response to F. gigantica infection, instead of serving an anti-inflammatory function. Our data also showed that the $R Q$ level of Foxp3 increased at $28 \mathrm{dpi}$. This level remained relatively high over the course of infection, which may have contributed to the Th1/Th2 balance in order to enhance parasite survival in the liver and to protect the host from overinflammation [59]. Foxp3-expressing Treg cells are critical to maintaining immune homeostasis by minimizing tissue pathology, while modulating host immune response against helminth infections $[60,61]$. The monitoring of Th17 and Treg cell offers a promising new perspective on the pathogenesis of F. gigantica infection and deserves further exploration.

\section{Conclusions}

This is the first study to characterize the correlation between the expression of Th1, Th2, Th17, and Treg cytokines and transcription factors with liver pathology in buffaloes, during the course of experimental F. gigantica infection. The expression patterns for the examined genes indicated that there were periods of differential regulation during $F$. gigantica infection, which may suggest either a mechanism of immune evasion based on modulation of transcription or a mechanism used by host tissue to limit the infection and tissue damage. Gene expression profiles showed a significant T-cell adaptive immune suppression between 3 and 10 dpi to facilitate parasite colonization and a more substantial transcript differential expression change (mixed Th1/ Th2 immune response) from 28 to $70 \mathrm{dpi}$, which may contribute to the parasite survival while minimizing host tissue damage. During late infection (98 dpi), the response was biased towards Th1/Treg in order to limit the host's Th2 protective response and promote chronic infection, which conincided with an increase in the number of recovered flukes and greater histopathological changes and fibrosis. Clearly, regulation of immune cytokine and transcription factor gene expression during F. gigantica infection is a complex mechanism involving a variety of parasite-specific and host-specific factors, perhaps depending on the parasite's needs for survival during various phases of infection. Research into the mechanisms governing differential expression of these immune-related genes, may shed light on the actual role of these immune regulatory factors in the pathogenesis of $F$. gigantica infection in buffaloes. Much remains to be elucidated about the role of various types of inflammatory responses, and the cross-talk between types of $\mathrm{T}$-helper responses, in fasciolosis.

\section{Abbreviations}

ANOVA: Analysis of variance; cDNA: Complementary deoxyribonucleic acid; Cq: Quantification cycle; DFd: Degrees of freedom denominator;

DFn: Degrees of freedom numerator; DNA: Deoxyribonucleic acid; DPI: Days post-infection; ELISA: Enzyme linked immunosorbent assay; EM: Encysted metacercariae; Foxp3: Forkhead box P3; GAPDH: Glyceraldethde-3-phosphate dehydrogenase; GATA: GATA binding protein; H\&E: Hematoxylin and eosin; IFN: Interferon; IL: Interleukin; ITS: Internal transcribed spacer; LSD: Least significant difference; mRNA: Messenger ribonucleic acid; qRTPCR: Quantitative reverse transcription-polymerase chain reaction; RBC: Red blood cells; rDNA: Ribosomal DNA; RNA: Ribonucleic acid; ROR: Retinoidrelated orphan nuclear receptor; RQ: Relative quantitation; SEM: Standard error of the mean; T-bet: T-box expressed in T cells; TGF: Transforming growth factor; Th: T helper; Treg: Regulatory T cell; WPI: Weeks post-infection

\section{Acknowledgements}

We are grateful to Xiao-Xuan Zhang, Wen-Bin Zheng and Jian-Gang Ma from Lanzhou Veterinary Research Institute, Chinese Academy of Agricultural Sciences, and Yang-Qun Kang, Xue-Fang Mei, Chang-Hong Hu, Yu Zhang, Yao-Yao Zhang, and Yi-Ying Liang from Guangxi University for their technical assistance.

\section{Funding}

Project financial support was provided by National Key Basic Research Program (973 Program) of China (Grant No. 2015CB150300) and by National Natural Science Foundation of China (Grant No. 31260605).

Availability of data and materials

The data supporting the findings of this article are included within the article.

\section{Authors' contributions}

WS, WYH and XQZ conceived and designed the study, and critically revised the paper. WS, ZYW, FKZ and KJL prepared the experimental samples. WS, 
ZYW and ZAS performed the experiments. WS, ZYW, FKZ and DYW analyzed the data. WS drafted the manuscript. HME helped in the data analysis and critical revision of the manuscript. All authors read and approved the final manuscript.

\section{Ethics approval}

The study design was reviewed and approved by the Animal Ethics Committee of Guangxi University. Animals used in the study were handled in accordance with good animal practices as required by the Animal Ethics Procedures and Guidelines of the People's Republic of China.

\section{Consent for publication}

Not applicable.

\section{Competing interests}

The authors declare that they have no competing interests.

\section{Publisher's Note}

Springer Nature remains neutral with regard to jurisdictional claims in published maps and institutional affiliations.

\section{Author details}

${ }^{1}$ College of Animal Science and Technology, Guangxi University, Nanning 530005, Guangxi Zhuang Autonomous Region, People's Republic of China. ${ }^{2}$ State Key Laboratory of Veterinary Etiological Biology, Key Laboratory of Veterinary Parasitology of Gansu Province, Lanzhou Veterinary Research Institute, Chinese Academy of Agricultural Sciences, Lanzhou 730046, Gansu Province, People's Republic of China. ${ }^{3}$ Faculty of Medicine and Health Sciences, School of Veterinary Medicine and Science, University of Nottingham, Sutton Bonington Campus, Loughborough LE12 5RD, UK. ${ }^{4}$ Jiangsu Co-innovation Center for the Prevention and Control of Important Animal Infectious Diseases and Zoonoses, Yangzhou University College of Veterinary Medicine, Yangzhou, Jiangsu Province 225009, People's Republic of China.

\section{Received: 22 August 2017 Accepted: 14 November 2017}

Published online: 08 December 2017

\section{References}

1. Mas-Coma S, Funatsu I, Bargues M. Fasciola hepatica and lymnaeid snails occurring at very high altitude in South America. Parasitology. 2001;123: $115-27$.

2. Cywinska A. Epidemiology of fascioliasis in human endemic areas. J Helminthol. 2005:79:207-16.

3. Yadav S, Sharma R, Kalicharan A, Mehra U, Dass R, Verma A. Primary experimental infection of riverine buffaloes with Fasciola gigantica. Vet Parasitol. 1999;82:285-96.

4. Ahmedullah F, Akbor M, Haider M, Hossain M, Khan M, Hossain M, et al. Pathological investigation of liver of the slaughtered buffaloes in Barisal district. Ban J Vet Med. 2007:5:81-5.

5. Raadsma H, Kingsford N, Spithill T, Piedrafita D. Host responses during experimental infection with Fasciola gigantica or Fasciola hepatica in merino sheep: I. Comparative immunological and plasma biochemical changes during early infection. Vet Parasitol. 2007;143:275-86.

6. Yuan W, Liu JM, Lu K, Li H, Duan MM, Feng JT, et al. Molecular identification and seasonal infections of species of Fasciola in ruminants from two provinces in China. J Helminthol. 2016;90:359-63.

7. Chai JY. Intestinal flukes. In: Murrell KD, Fried B, editors. Food-borne parasitic zoonoses. World class parasites, vol. 11. Boston: Springer; 2007. p. 53-115.

8. Zhang FK, Guo AJ, Hou JL, Sun MM, Sheng ZA, Zhang XX, et al. Serum levels of cytokines in water buffaloes experimentally infected with Fasciola gigantica. Vet Parasitol. 2017;244:97-101.

9. Dalton JP (Editor). Fasciolosis. Wallingford: CABI Publishing; 1999.

10. Mas-Coma S, Bargues MD, Valero MA. Fascioliasis and other plant-borne trematode zoonoses. Int J Parasitol. 2005;35:1255-78.

11. O'neill SM, Brady MT, Callanan JJ, Mulcahy G, Joyce P, Mills KH, et al. Fasciola hepatica infection downregulates Th1 responses in mice. Parasite Immunol. 2000;22:147-55

12. Zhang W, Moreau E, Hope J, Howard C, Huang W, Chauvin A. Fasciola hepatica and Fasciola gigantica: comparison of cellular response to experimental infection in sheep. Exp Parasitol. 2005;111:154-9.
13. Sallusto $F$, Lanzavecchia $A$. Heterogeneity of $\mathrm{CD}^{+}$memory $\mathrm{T}$ cells: functional modules for tailored immunity. Eur J Immunol. 2009;39:2076-82.

14. Infante-Duarte C, Kamradt T. Th1/Th2 balance in infection. Semin Immunopathol. 1999:21:317-38.

15. Zhang WY, Moreau E, Yang BZ, Li ZQ, Hope JC, Howard CJ, et al. Humoral and cellular immune responses to Fasciola gigantica experimental infection in buffaloes. Res Vet Sci. 2006;80:299-307.

16. Zhang FK, Zhang XX, Elsheikha HM, He JJ, Sheng ZA, Zheng WB, et al. Transcriptomic responses of water buffalo liver to infection with the digenetic fluke Fasciola gigantica. Parasit Vectors. 2017;10:56.

17. Huang $W Y$, He $B$, Wang $C R$, Zhu XQ. Characterisation of Fasciola species from mainland China by ITS-2 ribosomal DNA sequence. Vet Parasitol. 2004;120:75-83.

18. Schmittgen TD, Livak KJ. Analyzing real-time PCR data by the comparative CT method. Nat Protoc. 2008;3:1101-8.

19. Molina EC, Skerratt LF. Cellular and humoral responses in liver of cattle and buffaloes infected with a single dose of Fasciola gigantica. Vet Parasitol. 2005:131:157-63.

20. Shaikh AA, Bilqees F, Khan MM. Bile duct hyperplasia and associated abnormalities in the buffaloes infected with Fasciola gigantica. Pak J Zool. 2004;36:231-8.

21. Okaiyeto SO, Salami OS, Dnbirni SA, Allam L, Onoja II. Clinical, gross and histopathological changes associated with chronic fasciolosis infection in a dairy farm. J Vet Adv. 2012;2:444-8.

22. Marcos LA, Yi P, Machicado A, Andrade R, Samalvides F, Sánchez J, et al. Hepatic fibrosis and Fasciola hepatica infection in cattle. J Helminthol. 2007;81:381-6.

23. Grange D, Dhumeaux D, Couzineau P, Bismuth H, Bader J-P. Hepatic calcification due to Fasciola gigantica. Arch Surg. 1974;108:113-5.

24. Gironènes N, Valero MA, García-Bodelón MA, Chico-Calero I, Punzón C, Presno M, Mas-Coma S. Immune suppression in advanced chronic fascioliasis: an experimental study in a rat model. J Infect Dis. 2007;195:1504-12.

25. Rodríguez E, Noya V, Cervi L, Chiribao ML, Brossard N, Chiale C, et al. Glycans from Fasciola hepatica modulate the host immune response and TLR-induced maturation of dendritic cells. PLoS Negl Trop Dis. 2015;9:e0004234.

26. Pasare C, Medzhitov R. Toll pathway-dependent blockade of $\mathrm{CD}^{+} \mathrm{CD}_{25} 5^{+} \mathrm{T}$ cell-mediated suppression by dendritic cells. Science. 2003;299:1033-6.

27. Mangan PR, Harrington LE, O'quinn DB, Helms WS, Bullard DC, Elson CO, et al. Transforming growth factor- $\beta$ induces development of the $\mathrm{TH} 17$ lineage. Nature. 2006:441:231-4.

28. Sher A, Coffman RL. Regulation of immunity to parasites by $T$ cells and $T$ cell-derived cytokines. Annu Rev Immunol. 1992;10:385-409.

29. Swain SL, Weinberg AD, English M, Huston G. IL-4 directs the development of Th2-like helper effectors. J Immunol. 1990;145:3796-806.

30. Zhu J, Min B, Hu-Li J, Watson CJ, Grinberg A, Wang Q, et al. Conditional deletion of Gata3 shows its essential function in TH1-TH2 responses. Nat Immunol. 2004;5:1157.

31. Takeda K, Kamanaka M, Tanaka T, Kishimoto T, Akira S. Impaired IL-13mediated functions of macrophages in STAT6-deficient mice. J Immunol. 1996;157:3220-2.

32. Zhu J, Guo L, Watson CJ, Hu-Li J, Paul WE. Stat6 is necessary and sufficient for IL-4's role in Th2 differentiation and cell expansion. J Immunol. 2001;166:7276-81.

33. Mulcahy G, Dalton JP, Cathepsin L. Proteinases as vaccines against infection with Fasciola hepatica (liver fluke) in ruminants. Res Vet Sci. 2001;70:83-6.

34. Waldvogel AS, Lepage MF, Zakher A, Reichel MP, Eicher R, Heussler VT. Expression of interleukin 4, interleukin 4 splice variants and interferon gamma mRNA in calves experimentally infected with Fasciola hepatica. Vet Immunol Immunopathol. 2004;97:53-63.

35. Ingale SL, Singh P, Raina OK, Mehra UR, Verma AK, Gupta SC, et al. Interferon-gamma and interleukin-4 expression during Fasciola gigantica primary infection in crossbred bovine calves as determined by real-time PCR. Vet Parasitol. 2008;15:158-61.

36. Ingale SL, Singh P, Raina OK, Verma AK, Channappanavar R, Mehra UR. Interleukin-2 and interleukin-10 gene expression in calves experimentally infected with Fasciola gigantica. Livest Sci. 2010;131:141-3.

37. Dowling DJ, Hamilton CM, Donnelly S, La Course J, Brophy PM, Dalton J, et al. Major secretory antigens of the helminth Fasciola hepatica activate a suppressive dendritic cell phenotype that attenuates Th17 cells but fails to activate Th2 immune responses. Infect Immun. 2010;78:793-801.

38. Noya V, Brossard N, Rodríguez E, Dergan-Dylon LS, Carmona C, Rabinovich GA, et al. A mucin-like peptide from Fasciola hepatica instructs dendritic cells with parasite specific Th1-polarizing activity. Sci Rep. 2017;7:40615. 
39. Valero MA, Perez-Crespo I, Chillón-Marinas C, Khoubbane M, Quesada C, Reguera-Gomez M, et al. Fasciola hepatica reinfection potentiates a mixed Th1/Th2/Th17/Treg response and correlates with the clinical phenotypes of anemia. PLoS One. 2017:12:e0173456.

40. Lieberman LA, Cardillo F, Owyang AM, Rennick DM, Cua DJ, Kastelein RA, et al. IL-23 provides a limited mechanism of resistance to acute toxoplasmosis in the absence of IL-12. J Immunol. 2004;173:1887-93.

41. Yen D, Cheung J, Scheerens H, Poulet F, McClanahan T, Mckenzie B, et al. IL-23 is essential for T cell-mediated colitis and promotes inflammation via IL-17 and IL-6. J Clin Invest. 2006;116:1310-6.

42. Yang XO, Pappu BP, Nurieva R, Akimzhanov A, Kang HS, Chung Y, et al. T helper 17 lineage differentiation is programmed by orphan nuclear receptors RORa and RORY. Immunity. 2008;28:29-39.

43. Wan YY, Regulatory T. Cells: immune suppression and beyond. Cell Mol Immunol. 2010;7:204-10.

44. Kumar N, Raina OK, Nagar G, Prakash V, Jacob SS. Th1 and Th2 cytokine gene expression in primary infection and vaccination against Fasciola gigantica in buffaloes by real-time PCR. Parasitol Res. 2013;112:3561-8.

45. Allen JE, Sutherland TE. Host protective roles of type 2 immunity: parasite killing and tissue repair, flip sides of the same coin. Semin Immunol. 2014;26:329-40

46. Cortés A, Muñoz-Antoli C, Esteban JG, Toledo R. Th2 and Th1 responses: clear and hidden sides of immunity against intestinal helminths. Trends Parasitol. 2017;33:678-93.

47. Chakir H, Wang H, Lefebvre DE, Webb J, Scott FW. T-Bet/GATA-3 ratio as a measure of the Th1/Th2 cytokine profile in mixed cell populations: predominant role of GATA-3. J Immunol Methods. 2003;278:157-69.

48. Chantree P, Phatsara M, Meemon K, Chaichanasak P, Changklungmoa N, Kueakhai $P$, et al. Vaccine potential of recombinant cathepsin B against Fasciola gigantica. Exp Parasitol. 2013;135:102-9.

49. Molina EC. Serum interferon-gamma and interleukins-6 and-8 during infection with Fasciola gigantica in cattle and buffaloes. J Vet Sci. 2005;6:135-9.

50. Ilarregui JM, van Beelen AJ, Fehres CM, Bruijns SC, García-Vallejo JJ, van Kooyk Y. New roles for CD14 and IL- $\beta$ linking inflammatory dendritic cells to IL-17 production in memory CD4 ${ }^{+}$T cells. Immunol Cell Biol. 2016;94:907-16.

51. Donnelly S, O'Neill SM, Sekiya M, Mulcahy G, Dalton JP. Thioredoxin peroxidase secreted by Fasciola hepatica induces the alternative activation of macrophages. Infect Immun. 2005;73:166-73.

52. Flynn RJ, Mannion C, Golden O, Hacariz O, Mulcahy G. Experimental Fasciola hepatica infection alters responses to tests used for diagnosis of bovine tuberculosis. Infect Immun. 2007;75:1373-81.

53. Donnelly S, Stack CM, O'Neill SM, Sayed AA, Williams DL, Dalton JP. Helminth 2-Cys peroxiredoxin drives Th2 responses through a mechanism involving alternatively activated macrophages. FASEB J. 2008;22:4022-32.

54. Hacariz O, Sayers G, Flynn RJ, Lejeune A, Mulcahy G. IL-10 and TGF- $\beta 1$ are associated with variations in fluke burdens following experimental fasciolosis in sheep. Parasite Immunol. 2009;31:613-22.

55. Anderson CF, Oukka M, Kuchroo VJ, Sacks D. CD4 $4^{+}$CD25- Foxp3- Th1 cells are the source of IL-10-mediated immune suppression in chronic cutaneous leishmaniasis. J Exp Med. 2007;204:285-97.

56. Jankovic D, Kullberg MC, Feng CG, Goldszmid RS, Collazo CM, Wilson M, et al. Conventional T-bet ${ }^{+}$Foxp3 $^{-}$Th1 cells are the major source of hostprotective regulatory IL-10 during intracellular protozoan infection. J Exp Med. 2007;204:273-83.

57. Mahanty S, Mollis SN, Ravichandran M, Abrams JS, Kumaraswami V, Jayaraman $\mathrm{K}$, et al. High levels of spontaneous and parasite antigen-driven interleukin-10 production are associated with antigen-specific hyporesponsiveness in human lymphatic filariasis. J Infect Dis. 1996;173:769-72.

58. Anuradha R, Munisankar S, Dolla C, Kumaran P, Nutman TB, Babu S. Parasite antigen-specific regulation of Th1, Th2, and Th17 responses in Strongyloides stercoralis infection. J Immunol. 2015;195:2241.

59. Scalzo K, Plebanski M, Apostolopoulos V. Regulatory T-Cells: immunomodulators in health and disease. Curr Top Med Chem. 2006;6:1759-68.

60. Zhou S, Jin X, Chen X, Zhu J, Xu Z, Wang X, et al. Heat shock protein 60 in eggs specifically induces Tregs and reduces liver immunopathology in mice with schistosomiasis japonica. PLoS One. 2015;10:e0139133.

61. Escamilla A, Zafra R, Pérez J, McNeilly TN, Pacheco IL, Buffoni L, et al. Distribution of Foxp3 $3^{+} \mathrm{T}$ cells in the liver and hepatic lymph nodes of goats and sheep experimentally infected with Fasciola hepatica. Vet Parasitol. 2016;230:14.
62. Castillo-Velázquez U, Aranday-Cortés E, Gutiérrez-Pabello JA. Alternative activation modifies macrophage resistance to Mycobacterium bovis. Vet Microbiol. 2011;151:51-9.

63. Mingala CN, Konnai S, Venturina FA, Onuma M, Ohashi K. Quantification of water buffalo (Bubalus bubalis) cytokine expression in response to inactivated foot-and-mouth disease (FMD) vaccine. Res Vet Sci. 2009;87:213-7.

64. Puech C, Dedieu L, Chantal I, Rodrigues V. Design and evaluation of a unique SYBR green real-time RT-PCR assay for quantification of five major cytokines in cattle, sheep and goats. BMC Vet Res. 2015;11:65.

65. Pleasance J, Wiedosari E, Raadsma HW, Meeusen E, Piedrafita D. Resistance to liver fluke infection in the natural sheep host is correlated with a type-1 cytokine response. Parasite Immunol. 2011;33:495-505.

66. Maeda Y, Ohtsuka H, Tomioka M. Effect of progesterone on Th1/Th2/Th17 and regulatory $T$ cell-related genes in peripheral blood mononuclear cells during pregnancy in cows. Vet Res Commun. 2013;37:43-9.

\section{Submit your next manuscript to BioMed Central and we will help you at every step:}

- We accept pre-submission inquiries

- Our selector tool helps you to find the most relevant journal

- We provide round the clock customer support

- Convenient online submission

- Thorough peer review

- Inclusion in PubMed and all major indexing services

- Maximum visibility for your research

Submit your manuscript at www.biomedcentral.com/submit
Biomed Central 